La FMH a une longue tradition dans le traitement des données relatives à la démographie médicale. Celles-ci sont utiles aux sociétés de discipline médicale, aux sociétés cantonales de médecine, aux médecins et aux différentes organisations pour former leur opinion politique, forger leurs argumentations, planifier leurs besoins et réaliser leurs études. Le Département Données, Démographie et Qualité de la FMH propose jusqu'à la fin de l'année une série d'articles sur les sujets les plus demandés. Pour toute question ou évaluation plus détaillée, n'hésitez pas à vous adresser au département DDQ: ddq@fmh.ch / 0313591111.

\title{
Médecins en Suisse - Densité médicale et répartition régionale
}

Esther Kraft, Martina Hersperger

Correspondance:

Esther Kraft

FMH

Elfenstrasse 18

$\mathrm{CH}-3000$ Berne 15

Tél. 0313591111

esther.kraft@fmh.ch
Département DDQ

Fax 0313591112

\begin{abstract}
Changement de paradigme
Depuis que la statistique médicale est établie, le nombre de médecins en activité a régulièrement augmenté. Au début des années 90, cette évolution a incité certains milieux politiques à parler de «pléthore de médecins». D'autres ont vu une nette corrélation entre l'augmentation des coûts de la santé et l'augmentation de la densité médicale. Et bien d'autres encore ont attendu une véritable «invasion» de médecins étrangers suite à la libre circulation des personnes. Mais quelques années plus tard, une observation objective de la situation donne une toute autre image. Aujourd'hui, on constate une pénurie de médecins dans de nombreuses disciplines. Une étude de la KOF EPF Zurich [1] atteste que la densité médicale n'est pas un facteur déterminant dans l'augmentation des coûts de la santé, mais qu'elle y joue plutôt un rôle secondaire. L'évolution des coûts est due avant tout à d'autres facteurs, par exemple augmentation de l'espérance de vie ou progrès de la médecine. Les tableaux ci-après éclairent la situation actuelle des médecins en Suisse et présentent les évolutions principales.
\end{abstract}

Figure 1

Densité médicale en comparaison internationale.

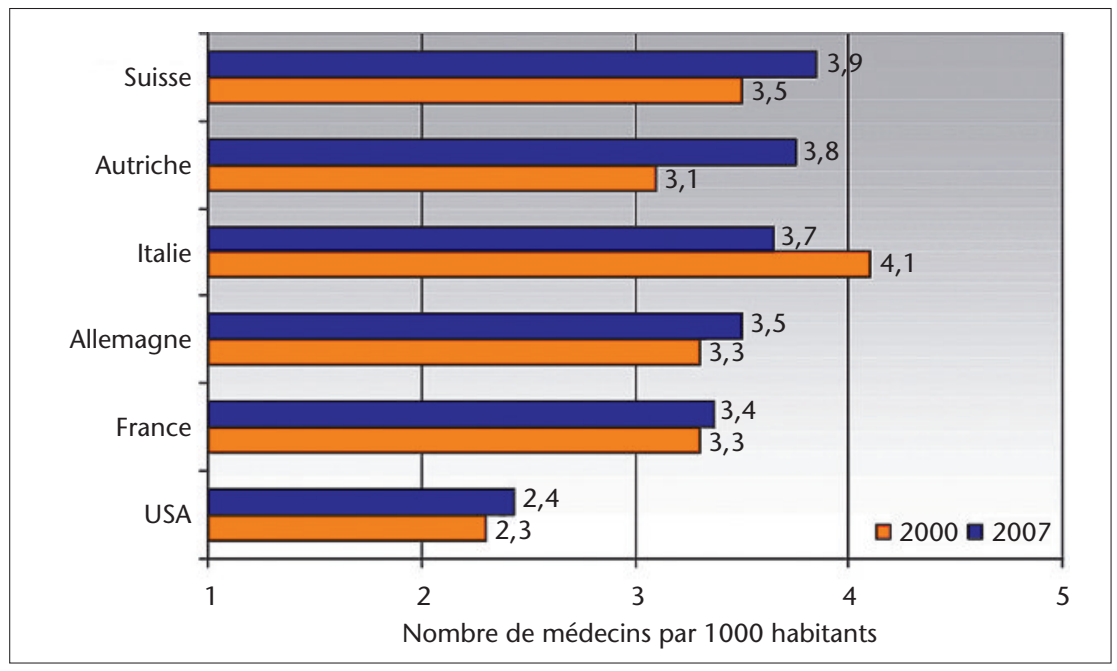

Densité médicale dans le secteur ambulatoire Selon l'OCDE [2], la densité des médecins exerçant en Suisse a passé de 3,5 à 3,9 par 1000 habitants entre 2000 et 2007. La comparaison internationale montre que la Suisse enregistre une des plus fortes densités médicales (cf. fig. 1). En 2008, cette densité a stagné à 3,9 , tous secteurs confondus.

Dans le secteur ambulatoire également, la densité médicale a augmenté. En 2000, elle était de 1,93 médecins par 1000 habitants; en 2008, elle a passé à 2,06 (augmentation d'env. 6.7\%). Les zones urbaines dotées d'un hôpital universitaire comme Zurich, Berne, Bâle, Genève et Lausanne comptent plus de médecins en activité que les régions rurales périphériques (cf. fig. 2). Les cantons de Suisse centrale ont une densité médicale plus basse. Les centres petits et moyens, axés sur une économie industrielle, tertiaire et touristique, se situent juste en dessous de la moyenne nationale [3].

Outre le canton d'Uri, la densité médicale a augmenté partout entre 2000 et 2008 , toutefois différemment d'un canton à l'autre. L'augmentation des médecins par 1000 habitants a été la plus forte (plus de 20\%) dans les cantons de Glaris et d'Appenzell (cf. fig. 3). C'est dans les cantons situés en dessous de la moyenne suisse que la densité médicale a augmenté le plus fortement ces huit dernières années, hormis les cantons de Schaffhouse, des Grisons, de Lucerne et d'Uri.

\section{Répartition géographique}

- La densité médicale n'est pas un facteur déterminant dans l'augmentation des coûts de la santé

- Dans les zones urbaines, la densité médicale est plus forte

- Dans les zones urbaines, la part des femmes est plus élevée et le taux d'occupation est plus bas en ce qui concerne les soins de premier recours

- La pénurie de médecins frappe différentes disciplines

- La couverture des soins est garantie grâce aussi aux médecins étrangers 
Entre 2000 et 2008, la densité médicale dans le secteur ambulatoire n'a pas seulement augmenté de manière différenciée dans les cantons, mais aussi dans les disciplines médicales (cf. fig. 4). Cette augmentation est nette pour la médecine générale et pour la psychiatrie et psychothérapie; elle a passé de 3,57 à 4,70 médecins généralistes par 10000 habitants $(+31 \%)$ et à 2,64 psychiatres et psychothérapeutes (37\%).

Ici aussi, l'on constate des différences cantonales et régionales (cf. fig. 5): En 2008, l'agglomération zurichoise enregistre la plus forte densité médicale avec 0,55 médecins porteurs du titre de spécialiste en médecine générale par 1000 habitants et le Tessin la plus faible avec 0,36 par 1000 habitants. La plus forte augmentation dans cette discipline concerne l'arc lémanique $(+44 \%)$ et la plus faible la Suisse orientale

Figure 2

Evolution de la densité médicale (nombre de médecins par 1000 habitants) dans le secteur ambulatoire entre 2000 et 2008 .

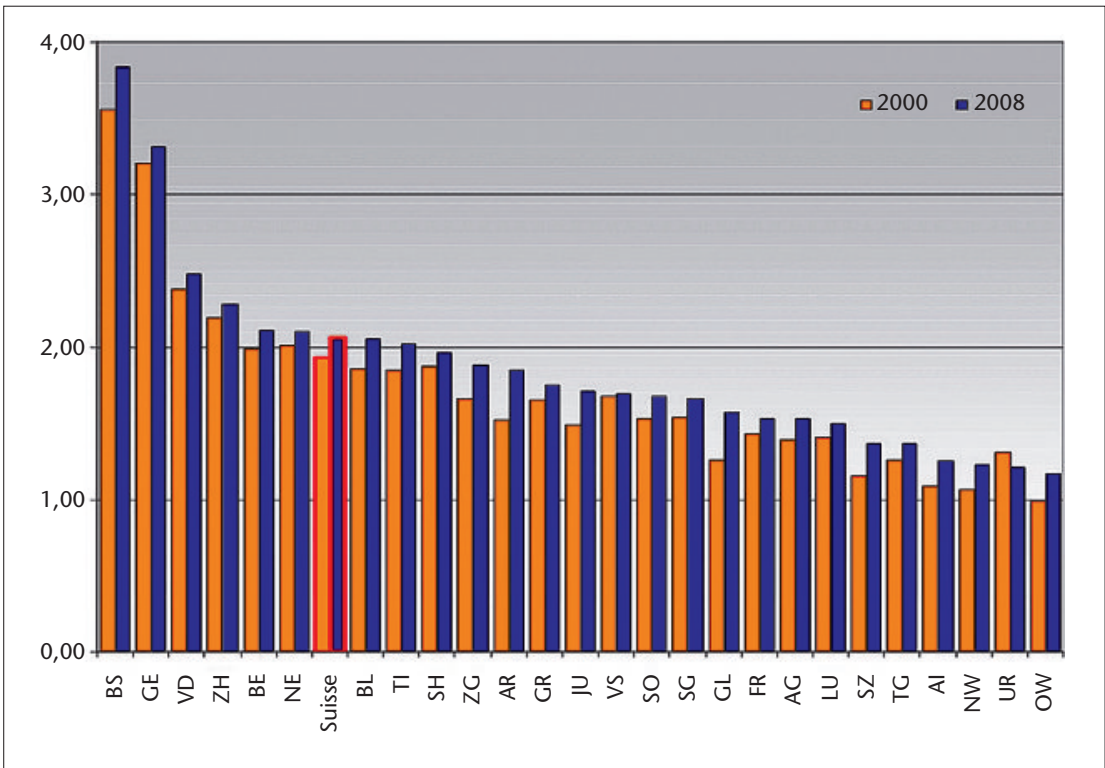

Figure 3

Evolution de la densité médicale (nombre de médecins par 1000 habitants) dans le secteur ambulatoire entre 2000 et 2008 .

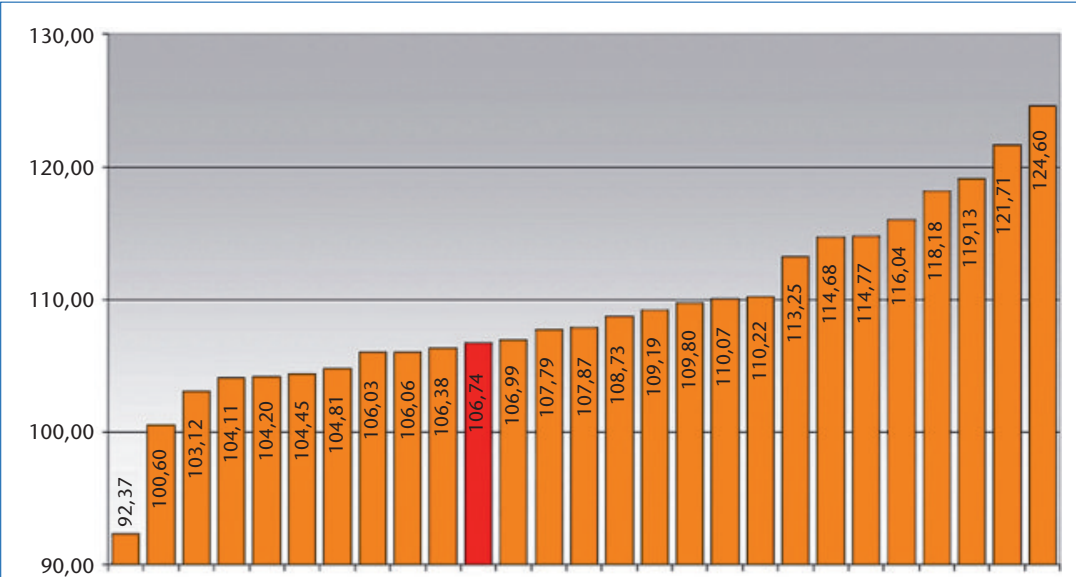

ऽ ऽ
(+19\%). Dans ce contexte, on comprend pourquoi les médecins de famille établis en zone rurale ont énormément de peine à trouver un successeur prêt à reprendre leur cabinet. La situation est différente pour la psychiatrie et la psychothérapie: ici, la densité médicale a augmenté d'env. $37 \%$ dans toute la Suisse. La Suisse centrale et la Suisse orientale enregistrent la densité de psychiatres la plus basse, mais c'est dans ces deux régions qu'elle a augmenté le plus fortement entre 2000 et 2008 (resp. $+71 \%$ et $+66 \%$ ). La médecine générale enregistre des différences cantonales et régionales moins importantes que la psychiatrie et psychothérapie, aussi bien par année que dans l'évolution en pourcentage sur plusieurs années.

\section{Perspectives}

A elle seule, la densité médicale ne permet cependant pas de faire la moindre constatation sur les prestations médicales effectivement prodiguées. L'augmentation du travail à temps partiel (due entre autres à la féminisation) doit être prise en compte dans cette interprétation. Dans une analyse qu'elle a faite de l'offre médicale ambulatoire en cabinet médical, l'Observatoire suisse de la santé (OBSAN) [4] a tenu compte du taux d'occupation et elle est parvenue à la conclusion que la fourniture de soins de premier recours est caractérisée dans les zones urbaines par une part plus élevée de femmes et un taux d'occupation plus bas, alors que dans les régions périphériques, c'est exactement le contraire (femmes moins nombreuses et taux d'occupation plus élevé). Dans cette étude, le taux d'activité est à nouveau une variable dérivée non basée sur les indications des médecins. Depuis qu'elle a révisé sa statistique médicale aux fins d'établir une base de données pertinente, la FMH dispose d'un outil permettant à chaque médecin d'indiquer son taux d'activité (cf. en cadré).

Dans le secteur hospitalier également, la pénurie de médecine semble être aigue. $\mathrm{H}+$, qui est l'organisation faittière des hôpitaux publics et privés, des cliniques et des institutions de soins de Suisse [5] met en garde contre une pénurie dramatique. H+ estime qu'il a manqué en Suisse, durant les années 2006 à 2008, près de 1170 médecins qu'il a fallu recruter à l'étranger. Les raisons de cette pénurie sont entre autres les suivantes:

- Exigences accrues envers les soins médicaux,

- Evolution démographique, vieillissement de la population et donc augmentation des maladies chroniques,

- Augmentation du nombre nécessaire de médecinsassistants et chefs de clinique suite à la réduction des horaires de travail

- Travail à temps partiel préféré par les femmes, toujours plus nombreuses à exercer la médecine.

Dans son étude [6], l'OBSAN souligne le rapport entre le vieillissement de la population et le recours aux prestations ambulatoires, en particulier dans la médecine générale. Elle note que cette discipline sera à 
Figure 4

l'avenir encore plus frappée par la pénurie de médecins. Les observations faites indiquent toutefois aussi qu'il faut faire la distinction entre la question de la densité médicale et celle du surplus de médecins versus pénurie de médecins. On constate que la Suisse dépend, aujourd'hui, déjà, de l'engagement de médecins étrangers dans différents domaines pour garantir la couverture des soins médicaux. La discussion sur les solutions possibles, par exemple la suppression du

Evolution de la densité médicale d'après certaines disciplines (nombre de médecins par 10000 habitants) dans le secteur ambulatoire entre 2000 et 2008.

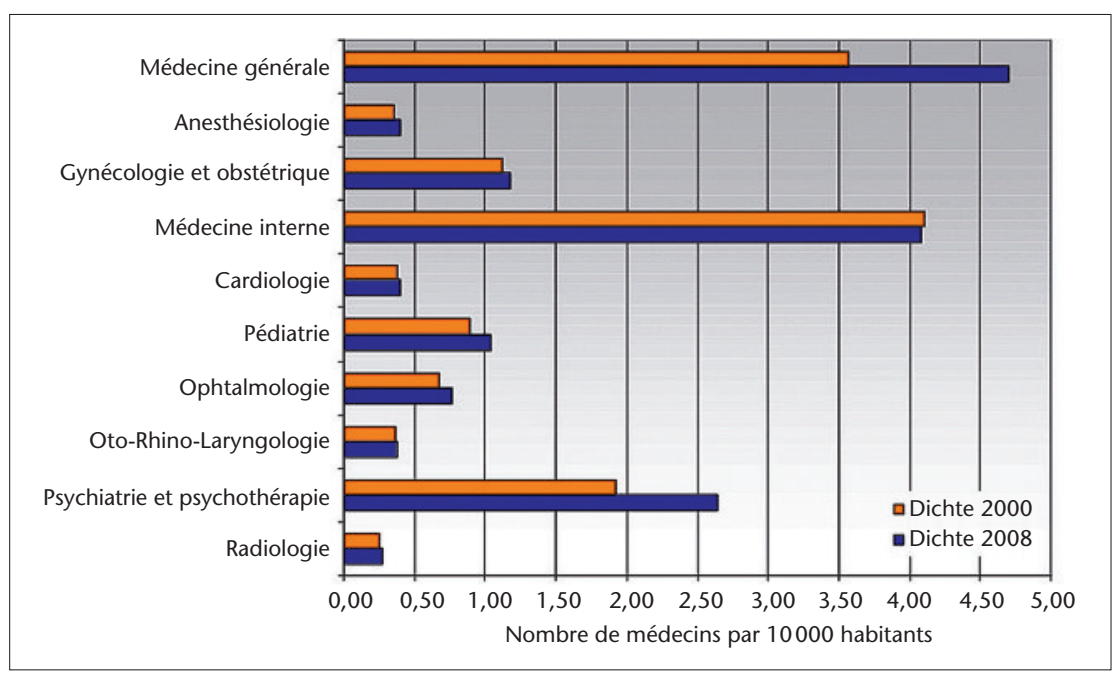

Figure 5

Densité médicale et sa modification entre 2000 et 2008 pour la médecine générale et pour la psychiatrie et psychothérapie.
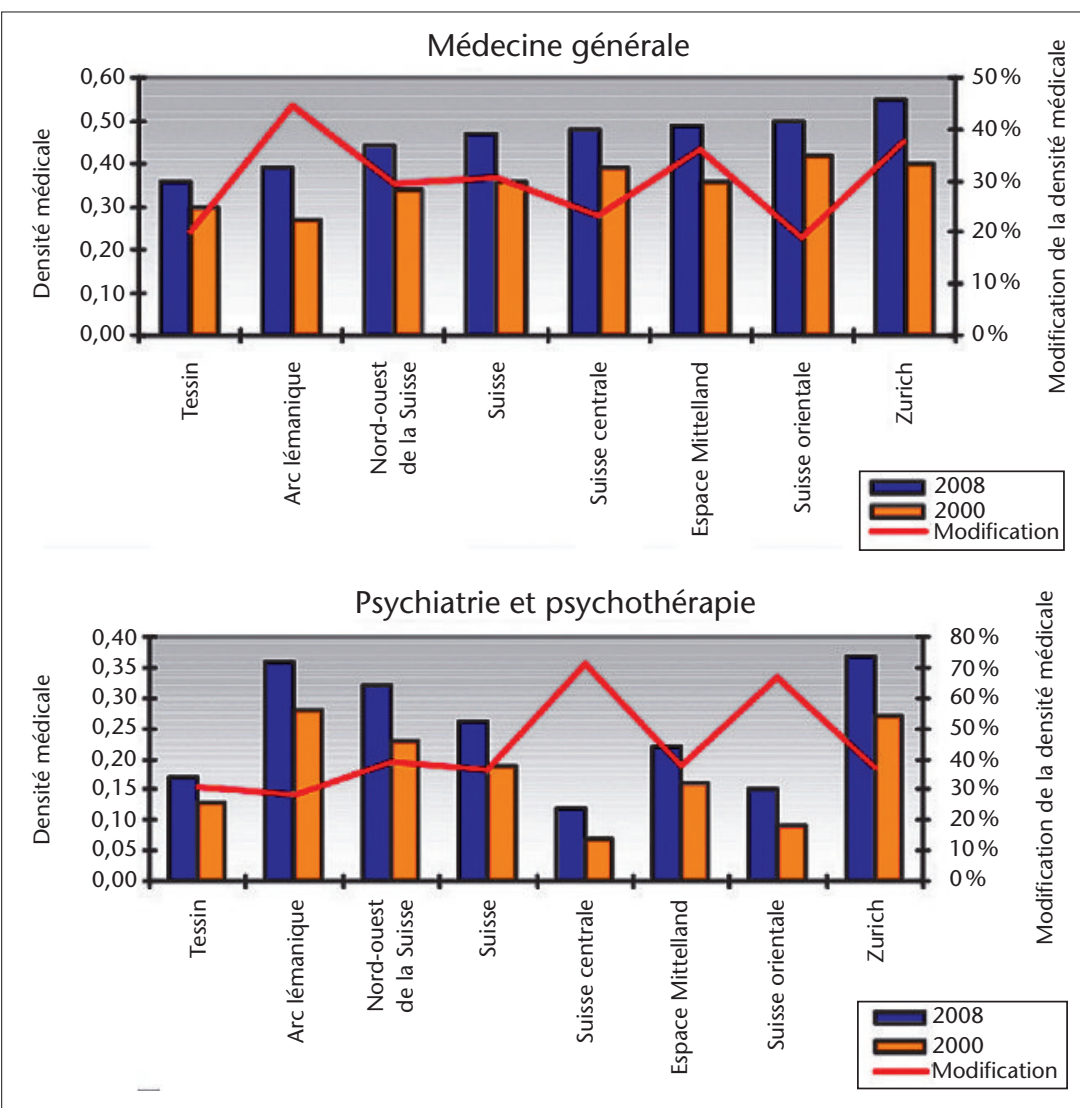

numerus clausus, la création de postes à temps partiel dans les hôpitaux, etc., va bon train.

Le prochain article de la série présentée dans le BMS «Données et démographie - informatif et passionnant» sera spécialement consacré à la formation postgraduée et continue. Ce $4^{e}$ article paraîtra le 4 novembre 2009 dans le $n^{\circ} 45 \mathrm{du}$ Bulletin des médecins suisses. Le premier article de cette série a été publié dans le $n^{\circ} 39$.

\section{Références}

1 Abrahamsen Y et al. Empirische Analyse des Gesundheitssystems Schweiz. Publikation der Konjunkturforschungsstelle (KOF) der ETH Zürich; 2005.

2 Eco-Santé OCDE 2009. Statistique et indicateurs pour 30 pays.

3 Médecins en cabinet privé (généralistes et spécialistes) pour 1000 habitants. www.obsandaten.ch/ indikatoren/5_1_1/2006/d/511.pdf; 23.10.2009

4 Jaccard Ruedin $\mathrm{H}$. et al. Offre et recours aux soins médicaux ambulatoires en Suisse, Document de travail. 22.2007;p. 25.

5 Communiqué de presse H+. Journée des hôpitaux 2009: Des métiers de tête et de cœur, Berne. www.hplus.ch/fileadmin/user_upload/Aktuell_Medien/ Medienkonferenzen/Nat. Spitaltag 2009/Deutsch/ 04_MM_Nationaler_Spitaltag_2009_D.pdf, 25.09.09

6 Seematter-Bagnoud L et al. Offre et recours aux soins médicaux ambulatoires en Suisse - Projections à l'horizon 2030; Document de travail 33. 2008; p. 20.

\section{La statistique médicale de la FMH:}

données de qualité grâce à vous!

Afin que la FMH puisse répondre aussi à l'avenir aux questions relatives à la démographie actuelle des médecins, la statistique révisée contient depuis 2008 également des précisions sur l'activité menée par les médecins.

En déclarant vos données, vous fournissez une contribution essentielle à une base de discussion et d'argumentation solide pour la régulation du corps médical.

Le nouveau questionnaire figure sur le portail internet www.myfmh.ch sous «Activité professionnelle, questions pour la statistique médicale de la $\mathrm{FMH}$ ».

Vous trouverez la statistique médicale de la FMH et les évaluations détaillées sous www.fmh.ch. Celle-ci est également présentée de manière compacte et claire dans notre dépliant disponible gratuitement (jusqu'à épuisement du stock) à l'adresse suivante: ddq@fmh.ch

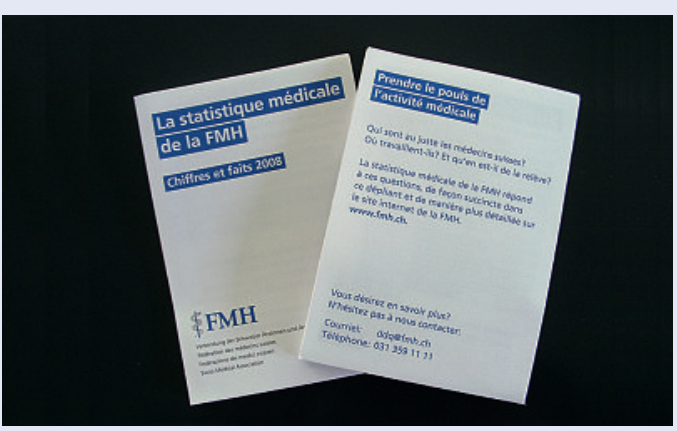

